\title{
Nutrition for Travel: From Jet lag To Catering
}

\author{
Shona L. Halson \\ Australian Catholic University \\ Louise M. Burke \\ Australian Institute of Sport and Australian Catholic University \\ Jeni Pearce \\ High Performance Sport New Zealand
}

\begin{abstract}
Domestic and international travel represents a regular challenge to high-performance track-and-field athletes, particularly when associated with the pressure of competition or the need to support specialized training (e.g., altitude or heat adaptation). Jet lag is a challenge for transmeridian travelers, while fatigue and alterations to gastrointestinal comfort are associated with many types of long-haul travel. Planning food and fluid intake that is appropriate to the travel itinerary may help to reduce problems. Resynchronization of the body clock is achieved principally through manipulation of zeitgebers, such as light exposure; more investigation of the effects of melatonin, caffeine, and the timing/composition of meals will allow clearer guidelines for their contribution to be prepared. At the destination, the athlete, the team management, and catering providers each play a role in achieving eating practices that support optimal performance and success in achieving the goals of the trip. Although the athlete is ultimately responsible for his or her nutrition plan, best practice by all parties will include pretrip consideration of risks around the quality, quantity, availability, and hygiene standards of the local food supply and the organization of strategies to deal with general travel nutrition challenges as well as issues that are specific to the area or the special needs of the group. Management of buffet-style eating, destination-appropriate protocols around food/water and personal hygiene, and arrangement of special food needs including access to appropriate nutritional support between the traditional " 3 meals a day" schedule should be part of the checklist.
\end{abstract}

Keywords: food hygiene, melatonin, traveler's diarrhea

Domestic and international travel is a common component of an elite athlete's yearly plan. Track-and-field athletes may travel to weekly competition in different countries during the main season (e.g., Diamond League), whereas distance runners and race walkers may complete less frequently in race series spread over several continents. National, regional, and World Championship competitions are held in different cities on annual/biennial schedules, while the Olympic Games held every 4 years typically involve reconnaissance trips and staging camps to familiarize athletes with the characteristics of the host environment. Meanwhile, specialized training camps targeting heat, altitude, or a dedicated training focus may also require international travel.

Strategies to manage travel fatigue and jet lag, and to ensure adequate hygiene and appropriate catering in the new environment, can minimize the challenges or deleterious effects of travel. Adjustment to the new location is particularly important when athletes have to compete soon after arriving, as jet lag, unaccustomed environmental conditions, illness, or inappropriate dietary intake has

Halson is with the School of Behavioral and Health Sciences, Australian Catholic University, Melbourne, Australia. Burke is with the Australian Institute of Sport, Belconnen, Australia; and the Mary MacKillop Institute for Health Research, Australian Catholic University, Melbourne, Australia. Pearce is with High Performance Sport New Zealand, Auckland, New Zealand. Address author correspondence to Shona L. Halson at shona.halson@acu.edu.au. the potential to significantly affect performance. However, lengthy periods of travel are challenging if new surroundings expose the athlete to suboptimal training or nutrition practices that interfere with the larger goals of the annual plan or key mesocycles of training. As the management of new or challenging environmental conditions (e.g., heat and altitude) is detailed elsewhere (Saunders et al., 2018), this review will focus on the challenges around travel itself, adaptation to new time zones, and meeting nutrition goals in a different/foreign food environment.

\section{Jet lag}

Jet lag, or circadian desynchronization, occurs due to a mismatch between the body's circadian rhythms and the external 24-hr lightdark cycle. This is the result of rapid transmeridian travel across multiple time zones and is influenced by both the number of time zones crossed and the direction of travel (Choy \& Salbu, 2011). Numerous symptoms are commonly experienced as a consequence of jet lag and have the potential to influence athlete performance negatively; these include fatigue, disturbed sleep, insomnia, hypersomnia, decreased alertness, headaches, mood disturbance, decreased motivation, appetite loss, and gastrointestinal distress (Choy \& Salbu, 2011; Forbes-Robertson et al., 2012; Kolla \& Auger, 2011).

There is evidence of a circadian rhythmicity for athletic performance, with time of day effects for various aspects such 
as strength, jump height and sprint, anaerobic and aerobic performance (Leatherwood \& Dragoo, 2013). Therefore, desynchronization caused by rapid air travel across multiple time zones has the potential to affect performance over a 24-hr period. Management strategies that alleviate the negative effects of jet lag are important for elite athletes, with the goals of realigning the circadian system as quickly as possible and minimizing the symptoms previously outlined. Zeitgebers are the rhythmic cues in the environment that synchronize the internal body clock to the external environment (Choy \& Salbu, 2011). Although the most powerful zeitgeber is light, there is some evidence of beneficial effects of nutritional interventions, such as melatonin, caffeine, and the manipulation of meal timing and composition.

\section{Melatonin}

Melatonin ( $N$-acetyl-5-methyoxytriptamine) is synthesized from serotonin in the pineal gland, with sleep being initiated when there is a concomitant rise in melatonin and a decline in body temperature (Choy \& Salbu, 2011). The effectiveness of exogenous melatonin is highly dependent on timing of administration: a phase advance in circadian rhythm occurs with intake in the afternoon or evening, whereas a phase delay occurs with intake in the early morning.

Most of the research on melatonin and jet lag has involved doses of 2-8 mg with eastward travel where a phase advance is required; the majority of such studies have demonstrated improved sleep and/or reduced daytime symptoms of jet lag (Choy \& Salbu, 2011). A recent systematic review reported that melatonin reduces subjective ratings of jet lag when compared with placebo; however, side effects such as hypnotic effects, confusion, and headaches may occur, and the risk of allergic reactions should be considered (Herxheimer, 2014).

Importantly, the regulation and availability of melatonin differs across countries. Melatonin is currently not regulated in the United States by the Food and Drug Administration. Furthermore, in countries such as parts of Europe, Australia, New Zealand, and the United Kingdom, melatonin is considered a medication and is available on prescription only; however, a nonprescription nutritional supplement form is readily available from pharmacies and health food stores and is not illegal to be in possession of melatonin. Indeed, because of its over the counter availability, particularly in the United States, melatonin is one of the most common forms of self-medication for sleep and jet lag. Formulations include liquids, tablets, and capsules that may contain other mixtures of vitamins, minerals, and herbal extracts (Erland et al., 2016). An investigation of 31 commercial melatonin products (purchased in Canada; however, as of 2017, melatonin is no longer available over the counter in Canada) found that melatonin content ranged from $18 \%$ to $478 \%$ of the labeled content, with a batch-to-batch variability of up to $465 \%$ (Erland et al., 2016). Furthermore, serotonin was identified in eight of the supplements. Therefore, although melatonin consumption is generally considered safe, as with many supplements, the lack of regulation should be considered with respect to both interaction with other medications and the possible presence of banned substances.

\section{Caffeine}

Caffeine antagonizes adenosine receptors in the brain, with normal dietary consumption sufficient to antagonize up to $50 \%$ in the inhibitory $\mathrm{A}_{1}$ and facilitatory $\mathrm{A}_{2 \mathrm{~A}}$ receptors (Landolt, 2015). About $200 \mathrm{mg}$ of caffeine has been shown to delay melatonin rhythm strongly and consistently by $40 \mathrm{~min}$ when taken $3 \mathrm{hr}$ before bedtime (Burke et al., 2015). Caffeine also increases cyclic adenosine monophosphate-dependent signaling and intracellular calcium release, both of which influence circadian timekeeping (Landolt, 2015).

Caffeine is one of the most common means of alleviating daytime fatigue experienced as a consequence of jet lag, with a number of studies examining its efficacy. A systematic review of individuals with jet lag or shift work disorder reported improvements in reasoning, memory, and attention when comparing caffeine with placebo (Ker et al., 2010). Furthermore, $300 \mathrm{mg}$ of slow-release caffeine taken at 08.00 am (destination time) for 5 days resulted in a more rapid resynchronization rate compared with placebo and similar results to melatonin supplementation (Pierard et al., 2001). In a further study by this group, caffeine decreased sleepiness in comparison with melatonin and placebo but resulted in more objective and subjective sleep complaints (Beaumont et al., 2004).

\section{Meal Timing}

The master clock controlling the human circadian system is in the suprachiasmatic nuclei in the anterior hypothalamus. The suprachiasmatic nuclei are primarily synchronized by the light/dark cycle; however, other primary cues exist for clocks located in the periphery (Potter et al., 2016). Feeding and fasting cycles are one of the primary time cues for the peripheral clocks; for example, the liver clock is particularly sensitive to both the timing and composition of food (Potter et al., 2016). Although meal timing has been proposed to increase synchronization following rapid air travel, with animal studies suggesting some support for this theory, few studies in humans have examined the effect of meal timing on jet lag. The Argonne diet (alternating days of high-protein intake with fasting/ low carbohydrate [CHO] foods, including a fast before arrival and a high-protein breakfast at the destination) may mimic the studies in animal models and the peripheral clock. The single study of this diet in military personnel found a reduction in jet lag symptoms (Reynolds \& Montgomery, 2002). However, due to the lack of research, a recent meta-analysis concluded that there is insufficient evidence to support the timing of meals as a means of alleviating jet lag (Herxheimer, 2014).

\section{Meal Composition}

Despite evidence around the effects of meal composition on sleep, specific investigation of its effects on jet lag or the circadian system is lacking. However, as disturbed sleep is a common and often debilitating consequence of jet lag, it is pertinent to examine recent findings in this area. A number of neurotransmitters associated with the sleep-wake cycle have been identified. These include serotonin (5-hydroxytryptamine), $\gamma$-aminobutyric acid, orexin, melaninconcentrating hormone, cholinergic, galanin, noradrenaline, and histamine (Halson, 2014). Nutritional interventions that act on these neurotransmitters in the brain may also influence sleep.

Dietary precursors can influence the rate of synthesis and function of a small number of neurotransmitters including serotonin that is subsequently converted to melatonin. Synthesis of 5-hydroxytryptamine is dependent on the availability of its precursor in the brain, the amino acid l-tryptophan (Trp). The Trp is transported across the blood-brain barrier by a system that shares other transporters including a number of large neutral amino acids (LNAA). Thus, the ratio of Trp/LNAA in the blood is crucial to the transport of Trp into the brain. Melatonin production may be 
increased by either increasing Trp intake or reducing the relative plasma concentration of LNAA. This can be achieved by several means including (a) dietary intake of proteins that contain more tryptophan than LNAA, (b) ingestion of CHO to facilitate the insulin-stimulated uptake of branched-chain amino acids into the muscle and increasing the ratio of free TRP to branched-chain amino acids, and (c) ingestion of a high-fat meal that may increase free fatty acids and result in increased free Trp, or (d) strenuous exercise causes mobilization of nonesterified fatty acids that compete with free tryptophan for binding to albumin; tryptophan is unique among amino acids in that it binds to albumin in the blood. (Halson, 2014).

Although investigating the effects of a high $\mathrm{CHO}$ versus highfat meal prior to sleep, Yajima et al. (2014) reported a decrease in slow-wave sleep (Stage 3 or deep sleep) in the first half of the night in the high-CHO trial. Afaghi et al. (2007) compared low- and high-Glycemic Index (GI) CHO-rich meals (eaten $4 \mathrm{hr}$ before bedtime) and a high-GI meal (1 hr prior) finding that the earlier consumed high-GI meal reduced sleep onset latency (time taken to fall asleep). In a second study (Afaghi et al., 2008), these authors compared a very low-CHO diet $(1 \% \mathrm{CHO}, 61 \%$ fat, and $38 \%$ protein) with an energy-matched control diet $(72 \% \mathrm{CHO}, 12.5 \%$ fat, and $15.5 \%$ protein), eaten $4 \mathrm{hr}$ prior to sleep. The very low$\mathrm{CHO}$ diet increased slow-wave sleep and all stages of nonrapid eye movement, whereas the control diet decreased rapid eye movement. This may be important for athletes, given that slow-wave sleep (which forms part of nonrapid eye movement sleep) is considered important for physical recovery. Rapid eye movement sleep is often considered important for mental recovery and is a period of sleep with low muscle tone and an increased propensity to dreaming.

Such results suggest that dietary intake prior to sleep has the potential to influence sleep architecture, with $\mathrm{CHO}$ composition and timing of meal intake being among the important variables. Brain tryptophan concentrations were found to be responsive to sequential ingestion of $\mathrm{CHO}$ and protein meals in rats (Fernstrom \& Fernstrom, 1995). Insufficient information currently prevents clear recommendations regarding meal composition and sleep; however, it is an area that has potential to mitigate some of the symptoms of jet lag. Further information on the role of macronutrients on sleep is found in recent reviews (Halson, 2014; St-Onge et al., 2016).

\section{Travel Fatigue and Eating En Route}

Even when travel does not involve transmeridian shifts, athletes may suffer fatigue due to interruptions to usual sleep, training, and eating routines while en route. The strategies previously described may be useful in re-establishing a new routine, while the degree of fatigue may be minimized by the careful selection of the mode, timing, and itinerary of travel, as well as behavior en route. The travel itinerary will influence practical opportunities to optimize meal type and timing during travel. Even short-haul travel can result in poor eating; packing food to consume on the trip reduces the opportunity for impromptu stops and the selection of less suitable choices from convenience options. When traveling by bus, food supplies should be in close proximity in appropriately chilled containers (i.e., not in the luggage bays). However, consideration of the problem of "boredom eating" might dictate the appropriate level of accessibility. Athletes who experience car (motion) sickness, with symptoms of nausea, vomiting, cold sweats, and headache, should seek medical advice, noting that some medications cause drowsiness and some over the counter products may contain prohibited substances. Nutritional recommendations include the avoidance of large meals, caffeine, and alcohol before and during travel and perhaps limiting intake where needed to simple foods, such as water and dry crackers. Females, children, migraine sufferers, and those on specific medications (including ibuprofen) appear to be more vulnerable to motion sickness; lying down, closing the eyes, or sucking lozenges sometimes offers relief.

Knowledge of flight duration and destination will determine the best approach to airline eating. Two meals may be provided during long-haul flights, with the first soon after departure and the second about $2 \mathrm{hr}$ before arrival. Athletes may need to carry appropriate snacks (bars, fresh and dried fruit, nuts) to supplement their intake, especially if they have higher energy needs. Food supplies should be appropriately accessible; for example, in hand luggage rather than in overhead bins when access is needed but not encouraging unnecessary "boredom eating." Due to international security limitations on liquids, drinks should generally be purchased/obtained once customs has been cleared. When traveling internationally, food from the country of origin and airline food must be left on board or declared at customs to avoid confiscation or heavy fines.

The timing, rather than the energy content, of meals may be associated with gut comfort, although small meals consumed before and during flight are reported to be better tolerated than larger meals (Armstrong, 2006; Loat \& Rodes, 1989). The pressure and dryness of cabin air increase insensible fluid losses (Leatherwood \& Dragoo, 2013); athletes should have adequate fluid available or request additional supplies from cabin staff to remain well hydrated. Of course, overhydration should be avoided, especially if it leads to interrupted sleep due to the need for toilet breaks. The diuretic effect of caffeine has been overstated in many travel guides and airline handouts; in fact, it typically has minimal effects on urine production in habitual consumers (Armstrong et al., 2005). Furthermore, if tea, coffee, and cola drinks are part of the everyday intakes of athletes, their sudden avoidance during a flight may lead to a reduced fluid intake and poorer hydration status and headaches due to caffeine withdrawal. Therefore, the intake of caffeinated beverages when traveling should be considered in terms of sleep management rather than hydration. Intake of alcohol is generally discouraged as its effects are magnified by the "altitude" of cabin air.

Recently, several airlines have reviewed the influence of atmospheric cabin pressure (typically equivalent to an altitude of $1,800-2,400 \mathrm{~m}$ above sea level) on taste sensation. Reports suggest that a $30 \%$ decrease in the sense of taste, particularly for salt and sweet; meanwhile sour, spicy, and bitter appeared unaffected, and seasonings including cardamom, lemongrass, and curry are perceived as more intense (Mayer et al., 2008). These changes are linked to a decreased sense of smell caused by filtered air and dried mucus membranes in the nasal passages as well as a reduction in the effectiveness of the olfactory and taste receptors due to the lower oxygen pressure. Research (Pellegrino et al., 2015) on 244 participants reported that $59 \%$ preferred eating while having a conversation (4\% preferred silence), especially for the dinner meal. Umami (the savory taste associated with seaweed and soy sauce) and the crunchiness/texture of food appear to be enhanced by "white" (background) noise, while salt and sweet tastes are reduced compared with eating in silence. Although this information is of interest due to its potential effects on food enjoyment and intake, studies have not been conducted in athletes and should include measurements of changes in eating behaviors.

Finally, changes in bowel habits are common with travel, due to dehydration, lack of movement, and alteration of the type and volume of food intake, including fiber intake. Constipation can be 
uncomfortable and may disrupt both the eating patterns and exercise patterns/performance of athletes on arrival at their destination. Athletes who are susceptible may wish to consider consuming additional fluids and foods that are fiber rich or have natural laxative properties (e.g., prunes, kiwifruit, or chia seeds) during long-haul travel. Constipation should be reported to the medical team; unsupervised use of laxatives is not recommended as these may be banned or contain banned substances.

\section{Travel Hygiene}

A twofold to threefold increase in the incidence of illness (including upper respiratory tract infection and gastrointestinal tract and infectious illness) has been reported with travel to foreign destinations with greater than a 5-hr time difference (Reilly et al., 2007; Schwellnus et al., 2012). In addition to alterations in immune health and illness resilience (Castell et al., 2018), the traveling athlete is at risk of exposure to new pathogens and perhaps an increase in scenarios of contact with many people in a confined area (e.g., dining halls, shared accommodation, transport, indoor competition venues). Illness while traveling can create a number of problems for the individual athlete and their team/entourage, ranging from failure to compete at peak form (or indeed at all) for the former, to distraction and wasted resources for the latter. There are few reliable data on the prevalence of illness during travel by athletes, although during some large sports competitions surveillance of illness and injury is undertaken. For example, among recent events, $5 \%$ of athletes who attended the Rio Olympic Games reported illness, with infectious illnesses being the most common problems $(56 \%)$; respiratory tract and digestive system concerns accounted for over two thirds of these (Soligard et al., 2017). No difference in the incidence of illness was noted between Rio 2016 and London 2012 Olympic Games (Soligard et al., 2017), but lower incidences were reported for the 2016 Paralympic Games (Derman et al., 2018). The 2017 World Athletics Championships in London experienced a norovirus problem in some accommodation venues necessitating quarantine restrictions on some athletes, which prevented them from competing (Kelner et al., 2017). An outbreak of norovirus also occurred during the 2018 Winter Olympics highlighting that concerns around hygiene are not restricted to warm weather events. In the United Kingdom, norovirus is regarded as a winter disease with increased warnings of risk leading into the winter weather. Although official reports and anecdotes around individual incidents are available, the true prevalence of illness during athlete travel is unknown, as athletes may fail to report, or ignore illness, fearing exclusion from competition or letting the team down.

Traveler's diarrhea is a common complaint on international trips (see Castell et al., 2018). Defined as three or more unformed bowel movements within $24 \mathrm{hr}$ with accompanying nausea, cramps, blood in stools, and vomiting; it is typically caused by contamination from fecal matter containing microbes (e.g., campylobacter, salmonella, Escherichia coli, Shigella), virus, and pathogens. Food or water contamination by $E$. coli is the most commonly reported cause (Patel et al., 2018). Although the duration of traveler's diarrhea is usually 3-4 days, with 1 day of severe incapacitation; in some cases, it can last for weeks and may lead to the development of irritable bowel in susceptible individuals. The associated dehydration and interrupted nutrition significantly affect an athlete's ability to train and compete; for detailed reviews, see Reilly et al. (2007) and Castell et al. (2018). The highly contagious aspect of infections involving the GI tract and viruses is an important consideration for its management.
Gastrointestinal distress and illness related to travel in some countries may be associated with poor hygiene practices of the athletes (especially hand hygiene practices) and the food safety and water practices/standards in the foreign location (Leatherwood \& Dragoo, 2013; Patel et al., 2018; Reily et al., 2007). Although some foods are inherently more at risk of contamination than others, a thorough review of studies of traveler's diarrhea found that avoiding such foods does not necessarily reduce illness prevalence and that poor hygiene practices in food service (e.g., restaurants and communal eating scenarios) were apparently more to blame than food selections or personal hygiene practices per se (Shlim, 2005). Probiotics appear to have a marginal protective role in reducing the incidence and severity diarrhea and upper respiratory tract infection (Berman et al., 2017). The effect appears is strain specific (Castell et al., 2018), particularly for upper respiratory tract infection (Berman et al., 2017). Evidence for prophylactic antibiotic use is unclear and needs to be balanced against disadvantages, including the presence of antibiotic resistant strains, the frequency of use for constant travelers, and side effects of some antibiotics on tendonopathy and rupture (Kohler, 2015). Use of rifaximin (antibiotic medication that halts the growth of bacteria) may be advised when necessary via medial support (Castell et al., 2018). Incidences of diarrhea should be reported to the medical team to enable the provision of relevant care, prevent cross-contamination of the team, and to investigate the cause. Antidiarrheal medications (e.g., Imodium) may be prescribed if the diarrhea causes incapacity. On return home, athletes who experience altered gut function should seek further medical advice. Diarrhea lasting over a week requires further investigation due to the possibility of parasite infestation, such as giardiasis.

The success of strategies targeted at preventing illness at elitelevel competitions is difficult to confirm. Hanstad et al. (2011) reported a lower prevalence of illness at the Vancouver Olympic Games among Norwegian athletes who had been provided with such strategies. However, there is a general lack of evidence around the impact of personal hygiene practices in preventing infectious illnesses (Shlim, 2005), despite the common sense of encouraging better behaviors. Team protocols around the prevention/management of illness should be developed prior to travel and updated on arrival at the destination once an inspection or assessment of outstanding issues around food safety has been completed. Strategies to prevent traveler's diarrhea and other infectious illnesses are summarized in Table 1, while reader is directed to other reviews (Castell et al., 2018) for advice on the management of traveler's diarrhea and gastrointestinal illnesses. The novice athlete may require more education and support around travel issues compared with the seasoned international traveler, depending on familiarization with the destination.

\section{Catering for Athletes in a New Environment}

A new location, even within the athlete's own country, inevitably leads to a change in the availability, type, and quality of food due to differences in the local food supply and catering arrangements. In some cases, the athlete will experience a reduction in these characteristics, creating challenges to the achievement of his or her specific nutrition plan. This can be critical for the success of the trip, especially during competition travel or specialized training camps (e.g., heat or altitude adaptation) where additional nutritional support is needed. The various causes of reduced quantity, quality, and accessibility of the food environment require different management strategies. For example, an athlete who has been 


\section{Table 1 Checklist for Reducing the Risk of Food Borne Illness}

1. Practice good personal hygiene-keeping hands away from the face, undertaking frequent hand washing with soap or hand sanitizers, and keeping water bottles and other personal equipment clean

2. Undertake research on travel hygiene issues that is destination specific and prepare a targeted plan for the trip

3. Drink safe potable water. Use sealed bottled water if there are any doubts and do not use ice unless from a safe source

4. Use local knowledge to identify best restaurant and catering locations

5. When possible, check food storage facilities and practices of catering venues (check for general cleanliness, look for overcrowding of storage, mixing of raw and cooked food on the same shelves, overflowing containers)

6. Take extra care with buffet-style catering. Request freshly prepared dishes rather than preprepared and reheated meals (including takeaways). Note practices around duration and temperature of food holding for both hot and cold foods. Be aware of cross-contamination with utensils and cutting boards/serving plates

7. Avoid local street food due to lack of knowledge about their food hygiene practices

8. If undertaking self-catering, take care with food handling and storage

9. In countries with different standards around food/water hygiene, take care (or avoid) high-risk foods

- Nonpotable water from the tap, including ice made from this water or foods that have been washed in it

- undercooked and raw meat, shellfish, seafood (including sushi)

- raw eggs

- unpasteurized dairy foods

- salads and raw vegetables

- fruit that cannot be peeled

- food that has been kept warm for an indeterminate amount of time

confronted with self-catering accommodation without adequate time, resources, or food preparation skills may need assistance in the form of predeparture cooking classes, a structured menu and ingredients, pairing with a partner who is proficient in self-management, or delivery of preprepared meals. Alternatively, many training and competition opportunities are situated in locations with a food supply that is limited or foreign to their home environment. Athletes may be reluctant to try new foods, be unsure

\section{Table 2 Challenges and Guidelines for Catering From the Perspective of the Caterer/Team Management}

Challenges
- Within limitations of budget, time-tabling constraints,
food supplies and cooking resources, the menu must
potentially cater for
O cultural, religious, and social preferences of athletes
and coaches, often from different backgrounds
O wide variation in the nutritional needs for different
events (e.g., shotput vs. marathon) and transitions
between training, tapering, competing, and
celebrating;
O opportunities to showcase the local cuisine of the
host city or country, and
O special needs for athlete with food allergies/
intolerances or other special nutritional
requirements.
- Hygiene practices must be robust to allow for the
potentially depressed immune systems of athletes
and the downsides of outbreaks of food-borne illness
(career upset for athlete and poor publicity for caterer).
- It can be hard to balance the number/variety within a
meal and across the menu cycle to avoid excess
choice (leading to overeating and extra expense) and
insufficient choice (preventing each individual from
achieving their needs).

\section{Solutions}

- Buffet-style or self-service food service is most suitable for feeding athletes because it offers the advantages of

$O$ fast service for athletes who are hungry and challenged for time

$\bigcirc$ maximum flexibility in allowing athletes to choose their food requirements

$O$ cost savings because of bulk cooking, reduced staffing requirements, and low food wastage.

- The number/variety of menu items may be determined by the size of the group and the duration of stay

$\bigcirc$ generally, the larger the group size, the greater the number of selections that may be needed/justified.

O lengthier stays require a longer menu cycle

- The menu should be dictated by nutritional needs and cultural preferences of the specific group. Depending on the specific training/event requirements, there may need to be additional focus on fuel needs, energy concerns (e.g., provision of low energy density options, portion control), or the provision of at-risk nutrients (e.g., calcium, iron).

- With large groups, special needs of some individuals (e.g., vegetarian, lactose-free choices) can generally be included within the basic menu. For smaller groups, special needs are better handled as an individual request.

- Special needs around allergies or food intolerances should be handled with care to provide appropriate choices and to avoid cross-contamination

- A buffet menu presented in large quantity serving dishes allows individuals to determine their own serving size. Foods that are expensive or considered "treats" can be portion controlled to encourage appropriate eating restraint.

- The menu should cater for between-meal snacks and exercise-related eating. If catering options are not open for extended hours, the menu should include portable food items that can be taken away for such snacks.

- It is useful to provide nutrition cards detailing the energy/nutrient/allergen profile of standard serves of menu food items - these should be available at the point of service to assist the athlete in making food choices. 


\section{Table 3 Challenges and Guidelines for Catering From the Athlete's Perspective}

\section{Challenges}

- Great quantities of food and reduced portion control or self-service may lead to overconsumption

- Many choices of food also lead to overconsumption and/or poor selections; buffet-style eating tends to promote a haphazard approach to meal choice

- Different and unusual foods. Some people are reluctant to try new foods and may not enjoy food that has been prepared differently to home

- Lack of supervision. Many young athletes are unused to the responsibility for their food intake.

- Distraction due to eating with large groups of people with different eating styles

- Anxiety about food availability. Eating with a large number of people or needing to line up for food can make the athlete anxious to take food in case it is not available later

- Eating for entertainment. Food provides an emotional and social role for athletes during times when training/energy needs may be reduced

- Food monotony. Although there may be plenty of variety within and between meals, after a while the experience of buffet/eating becomes repetitive

\section{Solutions}

- The athlete should know their nutritional goals and how to choose food to achieve these. It can be valuable to consult a sports dietitian/nutritionist for some specific advice.

- Surveying the food choices on the buffet and planning the meal before serving will assist with meeting nutrition goals.

- Seeking information from nutrition cards, education sheets or the chef/food manager can help with meal selection, especially for special needs (e.g., allergen-controlled meals)

- The athlete should choose a small and well-planned selection from the buffet; this helps to prevent overeating and promotes the perception of variety by leaving new choices for future meals

- Athletes should concentrate on their own needs and not concern themselves with the amount and type of food that others are consuming.

- Athletes should remove themselves from the food environment once they have finished eating to avoid exposure to the potential for boredom eating.

Note. Adapted from Practical sports nutrition (pp. 237-239), by L. Burke, 2007, Champaign, IL: Human Kinetics. Copyright 2007 by the Human Kinetics, Inc.

of equivalent options to their normal choices, or be limited to a narrower set of food options by considerations around water/ hygiene safety or the risk of contamination with banned substances (e.g., clenbuterol in meat) (Guddat et al., 2012). Sometimes, the general food environment is plentiful, but the athlete's financial resources or housing/catering arrangements create limitations; this is especially noticed when allocations do not cover the betweenmeal needs of athletes with high-energy requirements or goals of nutrition support around training/competition sessions. Common issues are a lack of fresh fruit and vegetables, items for special diets (e.g., gluten or allergen free, kosher/halal or vegan/vegetarian'; see Lis et al., 2018), portable/nonperishable snacks, and specialized sports foods/drinks. Management solutions include pretrip organization of required needs with the destination caterers or appropriate suppliers or the freighting/self-carriage of key foods from home within logistical considerations, such as luggage allowances, quarantine laws, and expense.

Nevertheless, some athletes may experience an increase in food availability, variety, and quality compared with their home scenario; for example, an athlete moving from a situation of food insecurity to an affluent environment, or any athlete experiencing the $24 \mathrm{hr}$, all you can eat, free-dining halls of the major sporting events (e.g., Olympic Games). This, especially when paired with a "holiday mentality," can lead to overeating or inappropriate eating,

\section{Table 4 Checklist for Travel}

1. Confirm the unique characteristics and challenges of the destination: This includes vaccination needs and risks of insect infestations (e.g., Zika, malaria); environment characteristics (altitude, heat, humidity, cold); catering arrangements; local food supply and eating customs; standards of food/water hygiene and safety; and quarantine regulations (food importing restrictions)

2. Know the special needs of athletes, coaches, and support staff: This includes medical conditions and food intolerances (e.g., coeliac); allergies (need for EpiPen and allergen notification in catering arrangements); and other special meal requirements (e.g., vegetarian, Kosher menus). Organize catering (airlines, hotels) well in advance

3. Consider nutritional needs during travel itineraries: Consider modes of transport, scheduled breaks, changes in time zones, plans for delays and canceled flights. Integrate a plan of provided meals, self-responsible meals, snacks, and fluids

4. Consider food culture and availability at the destination-staple foods and sources of key nutrients (e.g., protein and carbohydrate); important foods (e.g., fruit, vegetables, sports foods); special food needs (e.g., gluten free); cooking styles (addition of fat or spices); language issues (provide translation list of common items). Consider the risk of banned substances/contaminants in foods and manufactured products (e.g., clenbuterol in meats)

5. Identify the catering styles at the trip destination: Note the style of accommodation and meal service (e.g., shared rooms, in-room fridge, self-catering apartments, university halls of residence, hotel or external restaurants, buffet or à la carte meals, specialist dining halls—e.g., Olympic Village). Identify food availability at training and competition venues. Note timing of meal service and food availability, including access for those with extended training hours or other commitments (e.g., postevent drug tests). Locate supermarkets and fresh food stores to supplement organized catering. Bring additional food supplies (key foods or missing items) with the team if permissible/practical. Share food plans and challenges with the team if possible prior to departure

6. Establish team/group protocols around hygiene and food behavior: This includes use of hand sanitizers; behavior around self-catered meal or snack areas (e.g., blenders, woks, cooking utensils); policy about food in rooms; alcohol intake; and cleaning protocols for drink bottles

7. Undertake reconnaissance on arrival to finalize plan: Undertake a visual inspection of catering areas according to perceived risk (kitchens, food serveries and clean up areas, food storage and handling, food safety certificates including authenticity of produce). Update team hygiene plan if needed 
which can sabotage the goals of the trip in a different manner. There are numerous anecdotes about athletes gaining performanceimpairing amounts of body mass when unlimited food access, poor nutrition knowledge, and the reduced energy cost of tapered training coincide. Many problems around eating in a new location are shared to some degree by the individual, the team management, and the caterers. Thus, all can undertake preventive strategies before and during travel. Tables 2 and 3 identify some of the common challenges, with solutions that can be implemented by the caterer/ team management and athlete, respectively.

\section{Conclusions}

Appropriate management of challenges around jet lag, hygiene, and catering can support the athlete's goals while traveling. Planned intakes while en route and consideration of resynchronization of the body clock is useful, although more research on jet lag is needed. Management of food, water, and personal hygiene may help to reduce the risk of traveler's diarrhea and other infectious illnesses. Guidelines for catering, including appropriate behavior at buffet-style meals are needed to ensure that the food and water supplies are accessible, suitable, safe, and well used. A checklist of strategies is provided in Table 4.

\section{Acknowledgments}

All authors contributed to the writing and reviewing of this paper. No conflicts of interest are declared.

\section{References}

Afaghi, A., O'Connor, H., \& Chow, C.M. (2007). High-glycemic-index carbohydrate meals shorten sleep onset. The American Journal of Clinical Nutrition, 85(2), 426-430. PubMed ID: 17284739 doi:10. 1093/ajen/85.2.426

Afaghi, A., O'Connor, H., \& Chow, C.M. (2008). Acute effects of the very low carbohydrate diet on sleep indices. Nutritional Neuroscience, 11(4), 146-154. PubMed ID: 18681982 doi:10.1179/ $147683008 \times 301540$

Armstrong, L.E. (2006). Nutritional strategies for football: Countering heat, cold, high altitude and jet lag. Journal of Sports Sciences, 24, 723-740. PubMed ID: 16766501 doi:10.1080/02640410500482891

Armstrong, L.E., Pumerantz, A.C., Roti, M.W., Judelson, D.A., Watson, G., Dias, J.C., . . Kellogg, M. (2005). Fluid, electrolyte, and renal indices of hydration during 11 days of controlled caffeine consumption. International Journal of Sport Nutrition and Exercise Metabolism, 15(3), 252-265. PubMed ID: 16131696 doi:10.1123/ijsnem.15.3.252

Beaumont, M., Batejat, D., Pierard, C., Van Beers, P., Denis, J.B., Coste, O., . . . Lagarde, D. (2004). Caffeine or melatonin effects on sleep and sleepiness after rapid eastward transmeridian travel. Journal of Applied Physiology, 96(1), 50-58. PubMed ID: 12959951 doi:10.1152/ japplphysiol.00940.2002

Berman, S., Castell, L.M., Calder, P.C., Bishop, N.C., Blomstrand, E., Mooren, F.C., . . Nagatomi, R. (2017). Consensus statement: Immunonutrition and exercise. Exercise Immunology Review, 23, 8-50.

Burke, L. (2007). The communal dining hall: Perspectives from the athlete and caterer. In Practical sports nutrition (pp. 237-239). Champaign, IL: Human Kinetics.

Burke, T.M., Markwald, R.R., McHill, A.W., Chinoy, E.D., Snider, J.A., Bessman, S.C., . . W Wright, K.P., Jr. (2015). Effects of caffeine on the human circadian clock in vivo and in vitro. Science Translational
Medicine, 7(305), 305ra146. PubMed ID: 26378246 doi:10.1126/ scitranslmed.aac5125

Castell, L.M., Nieman, D.C., Bermon, S., \& Peeling, P. (2019). Exerciseinduced illness and inflammation: Can immunonutrition and iron help? International Journal of Sport Nutrition and Exercise Metabolism, 29(2). doi:10.1123/ijsnem.2019-0288

Choy, M., \& Salbu, R.L. (2011). Jet lag: Current and potential therapies. Pharmacy \& Therapeutics, 36(4), 221-231.

Derman, W., Schwellnus, M., Jordaan, E., Runciman, P., Blauwet, C., Webborn, N., . . . Stompjorst, J. (2018). Sport, sex and age increase risk of illness at the Rio 2016 Summer Paralympic Games: A prospective cohort study of 51198 athlete days. British Journal of Sports Medicine, 52, 17-23. PubMed ID: 29074477 doi:10.1136/ bjsports-2017-097962

Erland, L.A., Chattopadhyay, A., Jones, A.M., \& Saxena, P.K. (2016). Melatonin in plants and plant culture systems: Variability, stability and efficient quantification. Frontiers in Plant Science, 7, 1721. PubMed ID: 27899931 doi:10.3389/fpls.2016.01721

Fernstrom, M.H., \& Fernstrom, J.D. (1995). Brain tryptophan concentrations and serotonin synthesis remain responsive to food consumption after the ingestion of sequential meals. The American Journal of Clinical Nutrition, 61(2), 312-319. PubMed ID: 7840068 doi:10. 1093/ajen/61.2.312

Forbes-Robertson, S., Dudley, E., Vadgama, P., Cook, C., Drawer, S., \& Kilduff, L. (2012). Circadian disruption and remedial interventions: Effects and interventions for jet lag for athletic peak performance. Sports Medicine, 42(3), 185-208. PubMed ID: 22299812 doi:10. 2165/11596850-000000000-00000

Guddat, S., Fußhöller, G., Geyer, H., Thomas, A., Braun, H., Haenelt, N., . . Schänzer, W. (2012). Clenbuterol - Regional food contamination a possible source for inadvertent doping in sports. Drug Testing and Analysis, 4(6), 534-538. PubMed ID: 22447758 doi:10.1002/dta.1330

Halson, S.L. (2014). Sleep in elite athletes and nutritional interventions to enhance sleep. Sports Medicine, 44(Suppl. 1), 13-23. doi:10.1007/ s40279-014-0147-0

Hanstad, D., Ronsen, O., Andersen, S., Steffen, K., \& Engebretsen, L. (2011). For flight? Illnesses in the Norwegian team in the Vancouver Olympic Games. British Journal of Sports Medicine, 45, 571-575. PubMed ID: 21393259 doi:10.1136/bjsm.2010.081364

Herxheimer, A. (2014). Jet lag. BMJ Clinical Evidence, 2014, 2303. PubMed ID: 24780537

Kelner, K., Rawlinson, K., \& Gayle, D. (2017). London 2017: 30 People fall ill after norovirus hits athletics event. Retrieved from https:// www.theguardian.com/sport/2017/aug/08/london-2017-world-athelticschampionships-organisers-quarantine-hotel-gastroenteritis-outbreak

Ker, K., Edwards, P.J., Felix, L.M., Blackhall, K., \& Roberts, I. (2010). Caffeine for the prevention of injuries and errors in shift workers. Cochrane Database of Systematic Reviews, (5), CD008508. doi:10. 1002/14651858.CD008508

Kohler, R. (2015). Medical and nutritional issues for the travelling athlete. In L. Burke \& V. Deakin (Eds.), Clinical sports nutrition (5th ed., pp. 756-766). Sydney, Australia: McGraw Hill.

Kolla, B.P., \& Auger, R.R. (2011). Jet lag and shift work sleep disorders: How to help reset the internal clock. Cleveland Clinic Journal of Medicine, 78(10), 675-684. PubMed ID: 21968474 doi:10.3949/ccjm.78a.10083

Landolt, H.P. (2015). CIRCADIAN RHYTHMS. Caffeine, the circadian clock, and sleep. Science, 349(6254), 1289. PubMed ID: 26383940 doi:10.1126/science.aad2958

Leatherwood, W.E., \& Dragoo, J.L. (2013). Effect of airline travel on performance: A review of the literature. British Journal of Sports Medicine, 47(9), 561-567. PubMed ID: 23143931 doi:10.1136/ bjsports-2012-091449 
Lis, D.M., Kings, D., \& Larsen-Meyer, D.E. (2019). Dietary practices adopted by track-and-field athletes: Gluten-free, low FODMAP, vegetarian, and fasting. International Journal of Sport Nutrition and Exercise Metabolism, 29(2). doi:10.1123/ijsnem.2019-0309

Loat, C.E., \& Rodes, E.C. (1989). Jet lag and human performance. Sports Medicine, 8(4), 226-238. PubMed ID: 2692117 doi:10.2165/ 00007256-198908040-00003

Mayer, F., Burdack-Freitag, A., Breuer, K., \& Holm, A. (2008, August 17-22). Influence of low aircraft cabin pressure on taste and odor. Proceedings of the International Conference on Indoor Air 2008, Copenhagen, Denmark, Paper ID: 501.

Patel, A.R., Oheb, D., \& Zaslow, T.L. (2018). Gastrointestinal prophylaxis in sports medicine. Sports Health, 10, 152-155.

Pellegrino, R., Luckett, C., Shinn, S., Gude, K., Rhea, A., \& Seo, H. (2015). Effects of background sound on consumers' sensory discriminatory ability among foods. Food Quality and Preference, 43, 71-78. doi:10.1016/j.foodqual.2015.02.014

Pierard, C., Beaumont, M., Enslen, M., Chauffard, F., Tan, D.X., Reiter, R.J., . . . Lagarde, D. (2001). Resynchronization of hormonal rhythms after an eastbound flight in humans: effects of slow-release caffeine and melatonin. European Journal of Applied Physiology, 85(1-2), 144-150. PubMed ID: 11513308 doi:10.1007/s004210 100418

Potter, G.D., Cade, J.E., Grant, P.J., \& Hardie, L.J. (2016). Nutrition and the circadian system. British Journal of Nutrition, 116(3), 434-442. PubMed ID: 27221157 doi:10.1017/S0007114516002117

Reilly, T., Waterhouse, J., Burke, L., \& Alonso, J.M. (2007). Nutrition for travel. Journal of Sports Sciences, 25(Suppl. 1), S125-S134. doi:10. 1080/02640410701607445
Reynolds, N.C., Jr., \& Montgomery, R. (2002). Using the Argonne diet in jet lag prevention: Deployment of troops across nine time zones. Military Medicine, 167(6), 451-453. PubMed ID: 12099077 doi:10. 1093/milmed/167.6.451

Saunders, P.U., Garvican-Lewis, L.A., Chapman, R.F., \& Peeling, J.D. (2019). Special environments: Altitude and Heat International Journal of Sport Nutrition and Exercise Metabolism, 29(2). doi:10.1123/ ijsnem.2019-0256

Schwellnus, M., Derman, W., Jordaan, E., Page, T., Lambert, M.I., Readhead, C., . . . Webb, S. (2012). Elite Athletes travelling to international destinations $>5$ time zone differences from their home country have 2-3 fold increased risk of illness. British Journal of Sports Medicine, 46, 816-821. PubMed ID: 22875910 doi:10.1136/ bjsports-2012-091395

Shlim, D.R. (2005). Looking for evidence that personal hygiene precautions prevent traveler's diarrhea. Clinical Infectious Diseases, 41, S536-S540.

Soligard, T., Steffen, K., Palme, D., Alonso, J.M., Bahr, R., Lopes, A.D., . . . Engebretsen, L. (2017). Sports injury and illness incidence in the Rio de Janeiro 2016 Olympic Summer Games: A prospective study of 11274 athletes from 207 countries. British Journal of Sports Medicine, 51, 1265-1271. doi:10.1136/bjsports-2017-097956

St-Onge, M.P., Mikic, A., \& Pietrolungo, C.E. (2016). Effects of diet on sleep quality. Advances in Nutrition, 7(5), 938-949. PubMed ID: 27633109 doi:10.3945/an.116.012336

Yajima, K., Seya, T., Iwayama, K., Hibi, M., Hari, S., Nakashima, Y., . . . Tokuyama, K. (2014). Effects of nutrient composition of dinner on sleep architecture and energy metabolism during sleep. Journal of Nutritional Science and Vitaminology, 60(2), 114-121. PubMed ID: 24975221 doi:10.3177/jnsv.60.114 